\title{
Association of infection among intrauterine contraceptive device users in family planning clinics in Sudan
}

\author{
Mohammed Elkhair Bashir ${ }^{1 *}$; Awad Ali M. Alawad ${ }^{2}$ \\ ${ }^{1}$ Department of gynecology, Sharq alnile hospital, Sudan \\ ${ }^{2}$ Assistant Professor, Faculty of Medicine, University of Medical Sciences and Technology, Sudan \\ *Corresponding author E-mail: awadali82@hotmail.com
}

\begin{abstract}
Background: Intrauterine contraceptive device (IUCD) is one of the most widely used methods of reversible birth control. Consequently, the existence and consequences of insertion, removal and associated infection have received little attention

Objective: To identify the type and to determine the incidence of infection associated with IUCD users through screening by culturing endocervical swabs.

Methods: This is a descriptive, cross sectional, facility based study conducted in family planning clinics in Khartoum. This study was carried out on 35 ladies, attending the clinics during the period of study, using IUCD, presented for follow up, complaining or for removing the IUCD, who fulfilled the criteria of selection after obtaining an informed consent.

Results: The incidence of positive culture swab among users was $65.0 \%$. Among the study population, the age distribution was found to be varying between 24 and 39 years, taking a range of 4 years between groups of study population. Regarding complaint, vaginal discharge was found among $32(91.4 \%)$ users, intermenstrual bleeding was present among $15(42.9 \%)$ users, menorrhagia has been present in $11(31.4 \%)$ users, abdominal discomfort was noticed among $6(17.1 \%)$ users, and postcoital bleeding was present in $5(14.3 \%)$ users. Result of swab culture shows that, 12 women (34\%) of users culture of no growth, while 9 of them Staph.spp positive culture, there were 3 women (8.6\%) users with culture result of E.coli, and 3 women (8.6\%) users with culture result of candida albicans, there were 2 women $(5.7 \%)$ users with culture result of coliform and other 2 women $(5.7 \%)$ users with culture positive for trichomonas vaginalis.

Conclusion: The study concluded that incidence of positive cultures among IUCD users was $65.0 \%$ and about two third of female in the family planning clinics had mild to moderate levels of infection. The most common isolated organisms among IUCD users in this study were Staph spp, E.coli and Candida. The study also showed high rate of removal of IUCD copper type and most of removal due to dyspareunia.
\end{abstract}

Keywords: Intrauterine Contraceptive Device; Infection; Family Planning.

\section{Introduction}

IUD is one of the most popular contraceptive methods used worldwide; the most common type is Copper IUD. Different studies were conducted to explore the IUD related diseases particularly those associated with infection (Martinez \& Lopez-Arregui 2009). Some studies linked the infection-related disease to the insertion method and technique (Demirezen et al. 2005, Demirezen et al. 2006).

Reproductive tract infections (RTI) are common among women all over the world. They include three types; the" sexually transmitted disease, the endogenous infection caused by over-growth of organisms present in the genital tract such as bacterial vaginosis, and finally infections associated with medical procedures that manipulate the genital tract as unsafe abortion, pelvic examination, and IUD insertion (Bhurt et al. 1999).

Vaginal micro-flora is opportunistic bacteria, these bacteria as streptococci, staphylococcus, Escherichia-Coli, are vaginal habitat. Ohm, in his study classified them into two groups; the first is the predominant bacteria like Staphylococcus epidermidis, the other group is the potentially pathogenic, (Staphylococcus aurous, E-Coli, Streptococcus group A). The importance of studying the aerobic bacterial microorganism as E-Coli lies on fact that these organisms are opportunistic pathogens in the female genital tract and are associated in the pathogenesis of UTI (Demirezen et al. 2006). Also Staphylococcus aurous has its role in developing toxic shock syndrome. The aim of this study is to explore the presence of RTI symptoms among Sudanese women using IUD. The study also aims at finding a relationship between these symptoms and IUD use, duration, and type.

\section{Methods}

This is a cross-sectional facility based study. The study was conducted among family planning clinics in Khartoum state, in which the family planning services are provided. This study was carried out during the period from beginning of January to end of June 2013.

The study population were included all females who had IUCD, presented for follow up, complained or removed IUCD, in the above mention family planning clinics during the period of the study, selection is for all attendant, every day in the refer clinic for six months with the following exclusion criteria: Heavy blood during menstrual period at the time of sample collection, antibiotic use during previous 15 days and diabetic patients. A detailed and 
structured questionnaire was filled directly by the researchers, and so endocervical swabs from IUCD users were obtained used Dacron sterile swab under possible a septic technique, cultured in single well equipped laboratory, and according to the culture results, treatment was given to those patients whom were in need. Study variables include age, level of education, parity, occupation, duration of IUCD, type of IUCD, women's complaint, and result of cultured endocervical swab.

Data were entered into a Microsoft Excel spreadsheet and the statistical analysis was conducted using SPSS (Version 17). Statistical analysis was carried out using descriptive and analytical statistics. Simple frequencies and cross tabulation were done. Chi square test was used for proportions. P value of less than 0.05 was considered statistically significant.

The consent of the patients was obtained. A full explanation of the purposes and nature of the study was conveyed to them. The potential participants were clearly assured that their participation in this study is voluntary any data obtained would be treated confidentially and for the purpose of the research only.

\section{Results}

The age of study sample ranges between the 20-39 years old, 5 $(14.3 \%)$ of women in the age group $20-24$ years, in the age group 25 - 29 years we had $10(28.6 \%)$ women, in group from 30 -34 years we had $10(28.6 \%)$, and so for age group from $35-39$ years we had $10(28.6 \%)$ women. Parity less than three were 9 (25.7\%) women, parity between 3 and 5 were 19 (54.3\%) women, while parity more than 5 were 7 (20\%) women. Regarding level of education, There is two $(5.7 \%)$ illiterate women, we had $3(8.6 \%)$ women had primary education level, we had $27(77.1 \%)$ women had high secondary school education level, and we had $3(8.6 \%)$ women had university and post graduate education level. According to the types of IUDs, we had 32 (91.4\%) women used copper IUDs, we had $3(8.6 \%)$ women used Mirena.

According to the time since insertion of IUCD, we had 13 (37.1\%) women with insertion for one year, we had $5(14.3 \%)$ women with insertion for two years, we had $8(22.9 \%)$ women with insertion for three years, we had 4 (11.4\%) women with insertion for four years, we had $3(8.6 \%)$ women with insertion for five years, we had $2(5.7 \%)$ women with insertion for six years.

Table (1) represents distribution of study group according to the result of endocervical swab, we had $12(34.3 \%)$ women with culture result of no growth, we had $9(25.7 \%)$ women with culture result of staph spp, we had $3(8.6 \%)$ women with culture result of E coli, we had $3(8.6 \%)$ women with culture result of Candida albicans, we had $2(5.7 \%)$ women with culture result of coliform, we had $2(5.7 \%)$ women with culture result of trichomonas.

Table (2) shows distribution of study group according to association of dyspareunia and removed IUDs, we had 10 out of 12 removed IUDs were complaint dyspareunia, and 4 out of 23 were complaint and not removed ( $\mathrm{p}$ value o.ooo), all removed IUCDs are copper type 12 out of 35 IUCDs. Regarding complaint and type of IUCD used, we had $32(91.4 \%)$ women complained vaginal discharge all of them are copper devices users, we had 15 (42.9\%) women complained intermenstrual bleeding, 14(43.7\%) copper devices users, one (33\%) Mirena users, we had 11 (31.4\%) women complained menorrhagia, $10(31.3 \%)$ copper devices users, and one (33\%) Mirena users we had $6(17.1 \%)$ women complained abdominal discomfort, $4(12.5 \%)$ copper devices users and $2(66 \%)$ Mirena users, regarding women complained postcoital bleeding we had 5 (14.3\%), 4 (12.5\%) copper devices users, and one $(33 \%)$ Mirena users, there is one case out of 35 women reporting husband complaint.

We had $30(85.7 \%)$ women with color in the vaginal discharge, we had $24(68.6 \%)$ women with odour in the vaginal discharge, we had 14 (40\%) women complaint of dyspareunia, we had 13 $(37.1 \%)$ women complaint itching, we had 4 (11.4\%) women complaint soreness. According to the finding of speculum examination of the cervix, we had 7(20\%) women with ectropion, while the remainder was normal. The thread was seen in $32(91.4 \%)$ of users. and not seen in $3(8.6 \%)$ of users.

Table 1: Distribution of IUCD Users According To Culture of EndoCervical Swab

\begin{tabular}{lll}
\hline Result & Frequency & Percentage \\
\hline No growth & 12 & $34.3 \%$ \\
Staph spp isolated & 09 & $25.7 \%$ \\
Ecoli isolated & 03 & $08.6 \%$ \\
Candida albicans & 03 & $08.6 \%$ \\
Coliform isolated & 02 & $05.7 \%$ \\
Trichomonas vaginalis & 02 & $05.7 \%$ \\
Strepto spp isolated & 03 & $08.6 \%$ \\
Klebsellia isolated & 01 & $02.9 \%$ \\
Total & 35 & $100.0 \%$ \\
\hline
\end{tabular}

Table 2: IUCD Removed in Relation to Dyspareunia

\begin{tabular}{llll}
\hline & \multicolumn{3}{c}{ dyspareunia } \\
\hline IUCD removed & yes & no & Total \\
Yes & 10 & 2 & 12 \\
No & 4 & 19 & 23 \\
Total & 14 & 21 & 35 \\
\hline
\end{tabular}

\section{Discussion}

A total number of 35 women during their follow up in the family planning clinics were included in the present study, and the aims were to determine the incidence of IUCD associated infection, and to determine the most common organisms isolated in IUCD users presented for follow up, complaint or to remove it.

The impression from the result analysis concern age and parity distribution among the study group justified as, females involved in the marriage at early reproductive age that is why they have insertion at beginning of their life and so, part of them complete her family while still fertile and those why the IUCD still there for a long time that it may reach the menopause .most of the client and complaint patients had a copper type IUCD that may reflect the availability and cost effective in compared to Mirena type. The increased occurrence of births at older maternal ages is due to the increased population of women aged 35 to 40 , as well as later marriage, second marriage, the availability of better contraceptive options, and wider opportunities for further education and career advancement. Maternal education is one of the strongest predictors of use of IUCD, timing of childbearing, and the total number of children a woman will bear. Educated women tend to have low first birth rates when in their 20s, but higher first birth rates in their 30s, thus illustrating the trend of delayed childbearing related to educational achievement and career opportunities (Agarwal et al. 2004). However, older women also have a high prevalence of IUCD use resulting in unintended pregnancy. The influence of maternal age on the IUCD user rate was dependent of parity and education, although these characteristics were also minor factors for IUCD users. Women under age 25 have the lowest rate of IUCD user, housewives have the highest proportion of IUCD user and university and post graduate have the lowest rate of IUCD user.

Our study found that women during their follow up showed mild to moderate levels of IUCD associated infection, with the prevalence rate of positive culture swab about $65.0 \%$. While the rates about $3-5 \%$ of IUCD associated infection found in other studies of high-risk women $(13,14$, and 15). Also not correlate with other studies which states that the risk of infection with copper IUD is approximately 10 in 1,000 women characterized by abdominal pain, fever, vaginal bleeding or discharge or dyspareunia (Brezean et al. 2010, Buckley 1994, Siqueira et al. 2009). The most appropriate explanation for these differences may be due to, that other studies included high-risk women, while our study include all IUCD users women without identify whom at risk for infection . 
Also other studies were population based studies while our current study was hospital based study.

Twenty-three bacterial species and 19 genera were detected in vaginal secretions of the 35 healthy women, of which the highest concentrations were found for. Several Staph species as well as Candida albicans, coliform and trichomonas vaginalis were found in concentrations just below this limit. In previous studies the concentrations of bacterial species in vaginal secretions was reported to be approximately 100 times less than in the present study (Demirezen et al. 2005, Donders et al. 1991, Elhag et al. 1988, Harun et al. 1989). Staph spp, Ecoli, Candida albicans, coliform and trichomonas vaginalis were the most common organism isolated by endo cervical swab culture predisposing to IUCD associated infection in the present study, our finding not correlate with other studies which states that rare cases of staphylococcal and streptococcal attributed to IUD use have been reported (Gardien et al. 2001, Starcic Erjavec et al. 2007). These findings may have implications regarding complications associated with staphylococcal infections, beside that our literature reveals that, Staph. Aureus is responsible for many infections but it may also occur as commensal and the presence of S. aureus does not always indicate infection. Our study correlate with them in finding E.coli was one of the most common organisms associated with IUCD, in the current study in all age groups of women included in the study Mirena type IUCD, and through it affect in cervical mucosa acts like barrier, for sperm and so for microorganism that's what is achieved in our study, culture negative swab in all Mirena type IUCD users.

Vaginal discharge is one of most frequent gynecological problems encountered in females especially during their reproductive age; there for the present study find strong association between IUCD and vaginal discharge, these finding revealed that several species of microbes are responsible for vaginal infection among females. Of the organisms isolated, C. albicans had the highest isolation rate $(8.6 \%)$, followed by E. COLI $(8.6 \%)$ and klebsellia had the least isolation rate of $2.9 \%$.The high prevalent rate of $\mathrm{C}$. albicans observed in this study is an indication that $\mathrm{C}$. albicans is becoming a leading etiology of the reproductive tract infections among women using IUCD . The presence of S. aureus alongside with other bacteria such as E. coli, E. faecalis, Klebsiella spp, Proteus spp and P. aeruginosa may be attributed to immune status of the individual, lack of personal hygiene regarding the proximity of the vagina to the urethral tract from where these bacterial species may readily gain access into the vagina. Faecal organisms from the anus could also be introduced into the vagina during cleaning by using the ascending route (back to front) instead of the descending route (front to back).

It is not surprising to notice that most women included in the present study (91.4\%) were used cooper IUCD compared with Marina type, only (8.6\%) were used Marina, although the literature state that; the hormonal IUD Marina is considered to be more effective than other common forms of reversible contraception, such as the birth control pill, because it requires little to no action by the user after insertion (Argo \& Turnbull 2010, Hofmeyr et al 2010, Nohuz et al. 2012). The effectiveness of other forms of birth control is mitigated by the users themselves. If medication regimens for contraception are not followed precisely, the method becomes less effective. IUDs require no daily, weekly, or monthly regimen, so their typical use failure rate is therefore the same as their perfect use failure rate. This may be due to availability and low cost of cooper IUCD compared with Marina.

Limitation of our study is that prevention of IUCD associated infection was not evaluated. Association infection with IUCD still chelating, so all guide line encysted to follow infection prevention during insertion of IUCD in manners to prevent ascending infection(Guerreiro et al. 1998). Another limitation was that the study was used small sample size. Patient selection, sampling, intervention and data collection all done by the same observer. Furthermore, complaints of IUCD associated infection and its severity (mild, moderate, severe) is subjective rather than objective.

\section{Conclusions}

This study found the incidence of culture positive swab among IUCD users about $65.0 \%$ and about two third of female in the family planning refer clinics had mild to moderate levels of infection. The study showed a positive correlation between removal of IUCD and dyspareunia, type of IUCD removed. Among the organisms responsible for female genital discharge, Staff species followed by C. albicans and E. coli was found to be the highest microorganism isolated and it cut across all the clinical cases investigated during this study. Long-term use of the IUD is associated with impressive safety, especially Miren type, in users there is no growth of endocevical swab.

\section{References}

[1] Agarwal K, Sharma U \& Acharya V (2004): Microbial and cytopathological study of intrauterine contraceptive device users. Indian $J$ Med Sci 58, 394-399.

[2] Argo CM \& Turnbull EB (2010): The effect of intra-uterine devices on the reproductive physiology and behaviour of pony mares. Vet $J 186,39-46$. http://dx.doi.org/10.1016/j.tvj1.2009.07.013.

[3] Bhurt AW, Fikree FF, Bhurt AM, Channa GZ, Soomro RA \& Bhurt N (1999): Prevalence and risk factors of symptoms of pelvic inflammatory disease in a rural community of Jamshoro, Sindh, Pakistan. J Pak Med Assoc 49, 188-194.

[4] Brezean I, Aldoescu S, Catrina E, Valcu M, Ionut I, Predescu G, Degeratu D \& Pantea I (2010): Pelvic and abdominal-wall actinomycotic infection by uterus gateway without genital lesions. Chirurgia (Bucur) 105, 123-125.

[5] Buckley CH (1994): The pathology of intra-uterine contraceptive devices. Curr Top Pathol 86, 307-330. http://dx.doi.org/10.1007/978-3-642-768460_8.

[6] Demirezen S, Dirlik OO \& Beksac MS (2005): The association of Candida infection with intrauterine contraceptive device. Cent Eur J Public Health 13, 32-34.

[7] Demirezen S, Kucuk a \& Beksac MS (2006): The association between copper containing IUCD and bacterial vaginosis. Cent Eur J Public Health $14,138-140$.

[8] Donders GG, Moerman P, Caudron J \& Van Assche FA (1991): Intrauterine Candida infection: a report of four infected fetusses from two mothers. Eur J Obstet Gynecol Reprod Biol 38, 233-238. http://dx.doi.org/10.1016/0028-2243 (91)90298-Y.

[9] Elhag KM, Bahar AM \& Mubarak AA (1988): The effect of a copper intrauterine contraceptive device on the microbial ecology of the female genital tract. J Med Microbiol 25, 245-251. http://dx.doi.org/10.1099/0022261525-4-245.

[10]Gardien E, Schlegel L, Gregory A, Tognelli S, Fremaux A \& Geslin P (2001): [Apropos of a case of Streptococcus pneumoniae salpingitis, epidemiology of female genital pneumococcal infections]. Pathol Biol (Paris 49, 124-127. http://dx.doi.org/10.1016/S0369-8114 (00)00016-X

[11] Guerreiro D, Gigante MA \& Teles LC (1998): Sexually transmitted diseases and reproductive tract infections among contraceptive users. Int $J$ Gynaecol Obstet 63 Suppl 1, S167-173. http://dx.doi.org/10.1016/S00207292 (98)00201-X

[12]Harun R, Nayar S \& Thum Yin L (1989): Bacteriological study of extracted IUCDs. Malays J Reprod Health 7, 131-137.

[13]Hofmeyr GJ, Singata M \& Lawrie TA (2010): Copper containing intrauterine devices versus depot progestogens for contraception. Cochrane Database Syst Rev, CD007043.

[14]Martinez F \& Lopez-Arregui E (2009): Infection risk and intrauterine devices. Acta Obstet Gynecol Scand 88, 246-250. http://dx.doi.org/10.1080/00016340802707473.

[15] Nohuz E, Battista R, Tamburro S, Leonenko M, Bayeh S, Varga J \& Mage $\mathrm{G}$ (2012): [A more and more painful intrauterine device where it is not enough to see the wires to exclude malposition!]. Gynecol Obstet Fertil.

[16] Siqueira AK, Ribeiro MG, Leite Dda S, Tiba MR, Moura C, Lopes MD, Prestes NC, Salerno T \& Silva AV (2009): Virulence factors in Escherichia coli strains isolated from urinary tract infection and pyometra cases and from feces of healthy dogs. Res Vet Sci 86, 206-210. http://dx.doi.org/10.1016/j.rvsc.2008.07.018.

[17]Starcic Erjavec M, Rijavec M, Krizan-Hergouth V, Fruth A \& Zgur-Bertok D (2007): Chloramphenicol- and tetracycline-resistant uropathogenic Escherichia coli (UPEC) exhibit reduced virulence potential. Int $J$ Antimicrob Agents $\quad 30,436-442$ http://dx.doi.org/10.1016/j.ijantimicag.2007.06.025. 